hep-th/9504090

\title{
MASSLESS BLACK HOLES AND CONIFOLDS IN STRING THEORY
}

\author{
Andrew Strominger \\ Department of Physics \\ University of California \\ Santa Barbara, CA 93206-9530 \\ andy@denali.physics.ucsb.edu
}

\begin{abstract}
Low-energy effective field theories arising from Calabi-Yau string compactifications are generically inconsistent or ill-defined at the classical level because of conifold singularities in the moduli space. It is shown, given a plausible assumption on the degeneracies of black hole states, that for type II theories this inconsistency can be cured by nonperturbative quantum effects: the singularities are resolved by the appearance of massless RamondRamond black holes. The Wilsonian effective action including these light black holes is smooth near the conifold, and the singularity is reproduced when they are integrated out. In order for a quantum effect to cure a classical inconsistency, it can not be suppressed by the usual string coupling $g_{s}$. It is shown how the required $g_{s}$ dependence arises as a result of the peculiar couplings of Ramond-Ramond gauge fields to the dilaton.
\end{abstract}




\section{Introduction}

In the last several years there has been spectacular progress in our understanding of Calabi-Yau compactifications of string theory. A fascinating and ubiquitous phenomenon, beautifully exemplified in [1], is the occurrence of conifold singularities in the moduli space of classical string vacua. These are typically real codimension two surfaces in the moduli space at which a dimension two or three submanifold of the Calabi-Yau space shrinks to zero size, and the curvature of the moduli space metric blows up. They arise in virtually every Calabi-Yau string compactification.

The mathematics of these singularities is well understood [2, 3, 3, 1, 4, 4, 5, 6], but the physics is not. Consider a time-dependent modulus field in a cosmological setting which is slowly rolling around in a generic fashion. Eventually it will run in to a conifold singularity. Low-energy effective field theory - as described by a a sigma model whose target is the moduli space - is then inadequate for continuing the evolution because the sigma model equations of motion become singular. Our current understanding of strings on Calabi-Yau spaces must therefore be regarded as seriously incomplete.

There are several reasons why it is important to understand the physical behavior of string theory near conifolds. One of these is that, in our efforts to connect string theory with reality, it is important to find generic features of string compactifications which might lead to model-independent predictions. Conifold singularities are certainly one of the most significant such features. Secondly, under some weak assumptions, a generic superpotential will have local (or possibly global) minima at the conifold singularities. It is thus quite possible that the theory is driven to the conifold after supersymmetry breaking. Since this point is outside the main emphasis of the present paper its demonstration is given in a brief appendix.

In this paper we endeavor to explain the physics of conifolds. Specifically we show that, in the context of a type II string 1 , the breakdown of low-energy effective field theory may arise from integrating out a field which becomes massless at the singularity. The quanta of this field are extremally-charged Ramond-Ramond (RR) black holes! The conifold singularity can be resolved by including this field in the low-energy effective action.

The consistency of our picture requires that there is one and only one supermultiplet which becomes massless at the singularity. Ideally one would like to derive this from a semiclassical analysis of the extremal black holes. Unfortunately the counting of black

1 The proper interpretation of conifolds for heterotic strings remains a mystery. 
hole states is a tricky problem, so the best we can do at present is give some plausibility arguments in section 4.2. The consistency of our overall picture is of course evidence for our assumption, but it is not ruled out that there is a completely different resolution of the conifold singularities with a different numbers of massless states. Indeed, evidence for a picture quite different than that described herein was given in [2].

At first it may seem surprising that classical black holes can become massless. However this phenomenon has an appealing explanation from a ten-dimensional perspective. The IIA (IIB) theory has extremal black twobrane (threebrane) solutions [7] whose mass is proportional their area. After Calabi-Yau compactification these may wrap around minimal two (three) surfaces and appear as four-dimensional black holes. As the area of the surfaces around which they wrap goes to zero, the corresponding black holes become massless.

The structure of the low-energy effective field theory described here is very similar to that found in [8] for $N=2$ Yang-Mills. This latter case involves conifold singularities which are resolved by the inclusion of massless BPS magnetic monopoles, rather than extremal black holes. A characteristic feature of both examples are monodromies which describe how the charges of a state transported around the singularity are shifted by an $S P(2 r ; Z)$ transformation, where $r$ depends on the dimension of the moduli space.

A puzzling difference between the two cases is as follows. In the Yang-Mills case the form of the singularity can be derived from a one-loop computation in the light monopole effective field theory, or equivalently as an instanton effect in the original theory. In our case it is possible to derive the form of the singularity by a one-loop computation in the effective field theory with light black holes, but we do not understand the analog of the instanton computation. As discussed in section 4.3, such an instanton computation may make sense in the context of an as-yet-unknown dual formulation of string theory in which fundamental strings are solitons.

It has long been speculated that black holes should be treated as elementary particles. In the present paper we argue that string theory requires this - in the sense that extremal black holes contribute to virtual quantum loops in a manner determined by their mass and charge - in order to have a consistent resolution of conifold singularities.

In addition to conifold singularities, the moduli space of string vacua can also contain orbifold-like singularities at which low-energy field theory is in danger of breaking down. It has been understood for some time (see for example [9]) that such singularities can be resolved by the appearance of extra light vector bosons associated with the singularity. 
However for example in $K 3$ compactification of a type II theory there are no such massless states in string perturbation theory. It was conjectured in [10,11, 12, 13, and verified by construction in [14] that such states exist non-perturbatively with the correct degeneracies as RR solitons. The fact that the solitons have spin one as required for a gauge boson is related to the $N=4$ supersymmetry. The resolution we propose here of conifold singularities is similar in spirit. The difference in the present case is that there is only $N=2$ supersymmetry, and the solitons have maximum spin one-half. They therefore produce conifold rather than orbifold singularities when they become massless.

A striking feature of both the $N=4$ and $N=2$ cases is that consistency of the low energy theory is rescued by the intervention of nonperturbative quantum effects which are independent of the usual string coupling $g_{s}$ and are thus even larger than the $e^{-1 / g_{s}}$ effects discussed in [15]. However the nature of these effects is quite unusual and very restricted in form. We shall see in 4.1 that these large effects are possible due to the peculiarities of RR gauge fields. Related observations were made in [11].

This paper is organized as follows. Section 2 briefly reviews some relevant aspects of conifolds and special geometry. Section 3 describes the black holes and their properties. In section 4 it is explained how they resolve the conifold singularity, and we conclude in section 5. The appendix discusses the behavior of a generic superpotential near a conifold.

\section{Review of Conifolds and Special Geometry}

This section contains a lightning review of some relevant aspects of conifolds [1] and special geometry [16, 17]. More complete discussion and references can be found in [1] - [6].

A Calabi-Yau space $X$ has $b_{3}$ topologically non-trivial three-surfaces. Poincare duality implies the existence of a fixed integral basis $A_{I}, B^{J} I, J=1, . . \frac{1}{2} b_{3}$ of surfaces with intersections

$$
A_{I} \cap B^{J}=-B^{J} \cap A_{I}=\delta_{I}^{J}, \quad A_{I} \cap A_{J}=B^{I} \cap B^{J}=0 .
$$

This basis is unique up to $S p\left(b_{3} ; Z\right)$ transformations, under which $\left(A_{I}, B^{J}\right)$ transforms as a vector and the intersection matrix (2.1) is preserved. A choice of complex structure on $X$ is characterized by the $b_{3}$ periods of the holomorphic three-form $\Omega$

$$
\begin{aligned}
F_{I} & =\int_{A_{I}} \Omega, \\
Z^{J} & =\int_{B^{J}} \Omega,
\end{aligned}
$$


with respect to the fixed basis (2.1). The periods $Z^{I}$ (or alternately $F_{I}$ ) can be used as projective coordinates on the moduli space $\mathcal{M}$ of complex structures on $X$. They are projective because complex rescaling of $\Omega$ does not correspond to a change in the complex structure. $\mathcal{M}$ is a special Kahler manifold, and the periods (2.2) are a projective section of an $S p\left(b_{3} ; Z\right)$ vector bundle over $\mathcal{M}$ [16, 17].

In general $\mathcal{M}$ may contain a complex codimension one submanifold, at which one of the periods, say $Z^{1}$, vanishes. When $Z^{1}=0$, the corresponding three-surface $B^{1}$ degenerates to zero size, and the Calabi-Yau space $X$ is a singular "conifold". Since the surface $Z^{1}=0$ is complex codimension one in $\mathcal{M}$, it can be encircled by a closed loop. If $X$ is transported about this loop there is no guarantee that the basis (2.1) will return to itself. Indeed there is a typical $S p\left(b_{3} ; Z\right)$ monodromyl

$$
\begin{aligned}
& Z^{1} \rightarrow Z^{1} \\
& F_{1} \rightarrow F_{1}+Z^{1}
\end{aligned}
$$

This implies that near $Z^{1}=0$

$$
F_{1}\left(Z^{1}\right) \sim \text { constant }+\frac{1}{2 \pi i} Z^{1} \ln Z^{1}
$$

while all the other periods are smooth and nonzero (for a simple degeneration).

The existence of a singularity in the metric $\mathcal{G}$ on $\mathcal{M}$ follows readily from equation (2.4) and the formula for $\mathcal{G}$ [18]:

$$
\mathcal{G}_{I \bar{J}}=\partial_{I} \partial_{\bar{J}} \mathcal{K}
$$

where

$$
\mathcal{K}=-\ln \left(i F_{I} \bar{Z}^{I}-i Z^{I} \bar{F}_{I}\right)
$$

Substituting (2.4) for $F_{I}$ one finds that for $Z^{1}$ near zero the metric diverges as

$$
\mathcal{G}_{1 \overline{1}} \sim \ln \left(Z^{1} \bar{Z}^{1}\right)
$$

It is easily checked that the distance to $Z^{1}=0$ as measured by $\mathcal{G}$ is finite and that the scalar curvature diverges there [1].

2 This is the mondromy found for the quintic conifold in [1] and appears to be generic. However there are more complicated types of conifolds with multiple degenerations and different monodromies. Further analysis willl be required to see if these can be resolved in a similar fashion. 
To specify a string vacuum one must also choose a complexified Kahler class $J+i B$ on $X$. This leads to $b_{2}$ additional complex moduli. Mirror symmetry exchanges these with the complex structure moduli, implying a parallel description in terms of periods of the complexified Kahler form which can be found in 4 . Of course the classical moduli space is just the complexified Kahler cone and has no conifold singularities. However string theory instructs us to correct for the effects of worldsheet instantons which wrap around topologically non-trivial two-surfaces. One then finds that the periods can vanish at points where the instanton corrections are large. This corresponds to a conifold singularity at which the quantum-corrected area of a two-surface is degenerating to zero. Note that these are quantum corrections on the worldsheet only, and that no string loop effects are included in this calculation.

\section{Extremal Black $p$-branes}

In this section we will argue that type II string theories compactified on a Calabi-Yau space contain black holes which become massless near a conifold.

\subsection{The Type IIB Theory}

Ten-dimensional type IIB string theory [19] contains a self-dual five-form field strength obeying

$$
\begin{aligned}
F & =* F, \\
d F & =0,
\end{aligned}
$$

when the other antisymmetric tensor fields in the theory vanish. There is an associated conserved charge

$$
Q\left(\Sigma_{5}\right)=\int_{\Sigma_{5}} F
$$

for every homology class of five-surfaces $\Sigma_{5}$. The charge $Q$ can be carried by a $3+1$ dimensional extended object, or threebrane enclosed by $\Sigma_{5}$. Extended black hole solutions carrying the charge $Q$ are given by [7]

$$
\begin{aligned}
d s^{2}= & -\left(1-r_{+}^{4} / r^{4}\right)\left(1-r_{-}^{4} / r^{4}\right)^{-1 / 2} d t^{2} \\
& +\frac{d r^{2}}{\left(1-r_{+}^{4} / r^{4}\right)\left(1-r_{-}^{4} / r^{4}\right)} \\
& +r^{2} d \Omega_{5}^{2}+\left(1-r_{-}^{4} / r^{4}\right)^{1 / 2} d x_{i} d x^{i}, \\
F= & Q\left(\epsilon_{5}+* \epsilon_{5}\right), \\
\phi= & \phi_{0},
\end{aligned}
$$


where $\phi$ is the dilaton, $\int_{S^{5}} \epsilon_{5}=1, x^{i}, i=7,8,9$ is a coordinate on the threebrane, and the inner and outer horizons $r_{ \pm}$are related to $Q$ by $Q=2 r_{+}^{2} r_{-}^{2} \cdot r_{+}$is a regular outer horizon and there is a singularity at $r_{-}$. Classically there is a solution for every value of $Q$, but we will assume that there is a quantization condition so that $Q$ takes the minimal value $Q=g_{5}$.

At the quantum level most of these solutions are unstable due to Hawking radiation. They will decay to the extremal limit at which $r_{+}=r_{-}$and the metric becomes

$$
\begin{aligned}
d s^{2}= & -\left(1-r_{+}^{4} / r^{4}\right)^{1 / 2} d t^{2} \\
& +\frac{d r^{2}}{\left(1-r_{+}^{4} / r^{4}\right)^{2}} \\
& +r^{2} d \Omega_{5}^{2}+\left(1-r_{+}^{4} / r^{4}\right)^{1 / 2} d x_{i} d x^{i},
\end{aligned}
$$

and the solution is supersymmetric [0],20]. A Bogolmonyi bound implies

$$
\text { Mass }=Q \times(3-\text { volume })
$$

Now consider compactification of the IIB theory from ten to four dimensions on a Calabi-Yau space $X$. The four-dimensional theory will have $h_{21} N=2$ vector multiplets whose scalars are the coordinates $Z^{I}$ on $\mathcal{M}$ with metric $\mathcal{G}$ of (2.5). The extremal threebrane can then wrap around one of the three-surfaces $A_{I}, B^{J}$ in $X$. Such configurations will appear as ordinary localized solitons or black holes in four dimensions. Quantiztion of RR charge implies

$$
\begin{aligned}
& \int_{A_{I} \times S^{2}} F=n_{I} g_{5}, \\
& \int_{B^{J} \times S^{2}} F=m^{J} g_{5},
\end{aligned}
$$

where $S^{2}$ is a spatial two-sphere surrounding the black hole. The integers $m^{J}$ and $n_{I}$ are four-dimensional electromagnetic charges associated with the $h_{21}$ vector multiplets and the graviphoton. One expects that the minimal energy configuration will saturate a BPS bound 3 . Up to an overall constant the unique Kahler and $S p\left(b_{3} ; Z\right)$ invariant formula for the BPS mass is [12]

$$
M=g_{5} e^{\mathcal{K} / 2}\left|m^{I} F_{I}-n_{I} Z^{I}\right|
$$

3 A closely related class of black hole solutions saturating BPS bounds appears in [21]. 
Of special interest is a threebrane with all charges equal to zero except $n_{1}=1$. Its mass is then

$$
M=g_{5} e^{\mathcal{K} / 2}\left|Z^{1}\right|
$$

which vanishes at the conifold $Z^{1}=0$. The reason for this can be heuristically understood as follows. Accordnig to (3.5) the mass is proportional the area of the minimal surface around which the threebrane wraps. Practically by definition a conifold is a point at which this minimal area vanishes.

\subsection{The Type IIA Theory}

The type IIA theory has a four-form RR field strength $F$. The associated charge is carried by twobrane solutions [7]. Calabi-Yau compactification on $X^{\prime}$ leads to $b_{2}$ vector multiplets whose scalars are again the coordinates on a special Kahler manifold $\mathcal{M}^{\prime}$. The twobranes can wrap around minimal two-surfaces of $\mathcal{M}^{\prime}$ to produce four-dimensional extremal black holes. These black holes will then become massless at the conifold singularities of $\mathcal{M}^{\prime}$ where the area of the minimal surface (corrected by worldsheet instantons) degenerates. Since mirror symmetry exchanges IIB compactification on $X$ with IIA compactification on the mirror of $X$ [22,23], it will also exchange threebranes with twobranes. Hence if $X^{\prime}$ is the mirror of $X$ the analysis of the black hole solutions reduces to that considered above.

While the mathematical analysis is equivalent, the physical picture is significantly different. In the IIA case the conifold singularity and the massless states are a result of large instanton corrections. This will figure in to the comparison of section 4.3 with recent quantum results [8] in $\mathrm{d}=4$ supersymmetric gauge theories.

\section{Resolving the Conifold Singularity}

In this section we explain, following a parallel discussion in [8], how the appearance of a massless black holes resolves the puzzle of conifold singularities mentioned in the introduction. We wish to understand the four-dimensional, $N=2$ supersymmetric, lowenergy effective action resulting from Calabi-Yau compactification of type IIB string theory. At generic points in the moduli space, the massless fields will consist of the graviton

multiplet, $h_{21}$ vector multiplets and $b_{2}+1$ hypermultiplets. We shall focus on the conifold singularities in the moduli space of the vector multiplet. 
Near a conifold at $Z^{1}=0$, the BPS state with $n_{1}=1$ and all other charges equal to zero has a mass which vanishes as in (3.8). This state carries the minimal charge with respect to the $U(1)$ gauge field which lies in a supermultiplet with the moduli field $Z^{1}$, and is itself part of a hypermultiplet [24]. The low-energy effective action involving only moduli fields must break down near $Z^{1}=0$ because this state becomes light and can be excited. It should be replaced by an effective field theory in which the kinetic term of the $Z^{1}$ modulus does not diverge and there is an additional charged hypermultiplet. This is basically identicall to a situation described in [8]. In the low energy Wilsonian effective action there are no logarithms in $F_{1}$. However the one loop beta function obtains a contribution from the light charged hypermultiplet. Since the gauge coupling is a derivative of $F_{1}$, this implies a one loop correction to $F_{1}$ after integrating out the black hole hypermultiplet. This leads to

$$
F_{1}\left(Z^{1}\right) \sim \operatorname{constant}+\frac{1}{2 \pi i} Z^{1} \ln Z^{1},
$$

in agreement with (2.4). Thus the conifold singularity in the metric at $Z^{1}=0$ is just the usual type of singularity produced by intergrating out massless charged fields.

\subsection{Classical vs. $g_{s} \rightarrow 0$ Limit}

There is something puzzling about the preceding comments. In [8] the singularity in the moduli space arises from nonperturbative quantum effects and is resolved by quantum loops of light monopoles. In our example the singularity arises in the classical moduli space. Naively it would seem impossible to resolve such a singularity by quantum loops of anything, since these should be suppressed as $g_{s}=e^{\phi}$ (where $\phi$ is the string dilaton) vanishes.

The surprising fact 5 is that non-perturbative quantum effects are not all suppressed as $g_{s} \rightarrow 0$. Usually we think that in string theory $\hbar$ appears in the combination $\hbar e^{2 \phi}$ because a genus $g$ surface is always accompanied by a factor of $e^{2(g-1) \phi}$. This suggests

4 A fascinating difference is that in [8] a complex torus with degenerating cycles was introduced as a mathematical artifact to solve the equations determining the moduli space. In our case both the analog of the torus - the Calabi-Yau manifold - and the analog of the cycles are not introduced just to solve the equations but are "really there".

5 Observations closely related to the following were made in [10,14,13] and especially [11]. 
that as $e^{\phi} \rightarrow 0$ quantum effects are turned off. This argument has a loophole in theories with RR fields. The classical action is most naturally written in the form

$$
S \sim \int\left(e^{-2 \phi} R+F^{2}+\ldots\right)
$$

where $F$ is a RR field strength. This seems to contradict the expectation from string perturbation theory that the classical action should be proportional to $e^{-2 \phi}$, but it is not a contradiction because [25,11] this factor could be reinstated in front of the second term by rescaling the potentials. However this would lead to peculiar gauge transformation laws and quantization conditions. There is no reason why nonperturbative effects associated with (4.2), which does not have a uniform overall factor of $1 / g_{s}^{2}$, should behave as $e^{-1 / g_{s}^{2}}$.

We wish to see that, after an appropriate rescaling, loop effects of RR black holes contain no factors of $g_{s}$. The fastest way to see this is to note that after rescaling the metric by $e^{-2 \phi}$, the four dimensional effective action at generic points in the moduli space can be written in the form 6

$$
S_{4} \sim \int d^{4} x \sqrt{-g}(R+(\text { vector multiplets })+(\text { hypermultiplets })+\ldots)
$$

The dilaton $\phi$ lives in a hypermultiplet. $N=2$ supersymmetry forbids neutral couplings between vector multiplets and hypermultiplets [16]. Thus the first two terms in the action are simply unaware of the value of $g_{s}$. The black holes which becomes massless at the conifold points are soliton solutions involving only these two terms and are therefore also unaware of the value of $g_{s}$. In a low energy effective field theory they will be represented by a hypermultiplet with a $g_{s}$-independent action. Therefore quantum loops of these solitons do not involve $g_{s}$.

In conclusion, the $g_{s} \rightarrow 0$ limit of the low-energy effective action of the full quantum theory is very different than the low-energy effective action at string tree-level. The latter is inconsistent because of conifold singularities. The inconsistency may be resolved by the inclusion of non-perturbative quantum effects.

\footnotetext{
6 These are the variables in which spacetime supersymmetry is manifest.

7 This implies a nonperturbative nonrenormalization theorem for the metric (2.5) 26].
} 


\subsection{Degeneracies of States}

A second puzzle concerns the number of states which become light near a conifold. The mass of a state with $n_{1}=2$ also vanishes. If this is interpreted as a charge-two, single-particle state, there would be an extra contribution to the beta function, ruining the agreement between (2.4) and (4.1). We therefore assume that this does not correspond to a single particle state. Rather it is a two-particle state consisting of two charge-one particles.

The counting of extremal black hole states is a long-standing unsolved problem in quantum gravity. We do not know how to derive our assumption from first principles (except from the requirement of a consistent low-energy theory), but it can be supported by consideration of some other examples. A first example is the 't Hooft-Polyakov monopole. The charge-two solution is just a point in the moduli space of two charge-one solutions, and there is no separate charge-two monopole. However black hole moduli spaces are less well defined near the coincident point and the question is not so easy to answer. In a second example [14], minimally charged string solitons were constructed as extremal black holes in a $K 3$ compactification of type IIA string theory. Closed loops of these solitons had just the right masses and degeneracies to provide the enhanced gauge symmetries at the points in moduli space where the $K 3$ surface degenerates. However the soliton string solution can carry any axion charge. If the charge-two string had been viewed as distinct from two charge-one strings, a consistent picture would not have emerged. A related example is a fundamental string solution 27,28], which has just the right charge/mass ratio to be viewed as an extremal black hole [7]. If we treated the multiply-charged solutions as distinct objects, there would be an infinite number of gravitons!

Other examples however seem to go against our assumption. In [11], the relation between type IIA string theory and $d=11$ supergravity seems to require an infinite tower of distinct multiply-charged states. Also the spectrum of Dabohlkar-Harvey winding states [27,28 of heterotic string theory at charge two contains both a two-string state and a doubly-wound, one-string "bound state at threshold". The higher $n_{1}$ states discussed here might be viewed as a similar type of winding state.

To summarize, consistency of the low energy theory seems to require that ther are no multiply-charged single-particle states. It would be interesting to verify (or disprove!) this from some other starting point, such as possibly a semiclassical analysis of the solutions and their moduli spaces. 


\subsection{Quantum Duality}

A further puzzle concerns the fact that the conifold singularities discussed herein arise in what is usually referred to as classical string theory, as opposed to the conifold singularities of $N=2, d=4$ Yang-Mills [8], which are not present classically but arise from quantum instanton corrections. This seems to be a radical difference. However there have been conjectures [28, 27, 29, 25, 30, 31, 11] that there exists a dual formulation of string theory in which fundamental strings arise as solitons. In any such dual formulation of string theory, a worldsheet instanton of the ordinary formulation of string theory will be a spacetime instanton leading to nonperturbative quantum effects. In type IIA string theory, the conifold singularities are produced by large effects from worldsheet instantons. In a dual formulation of the IIA string, the conifold singularities would then be interpreted as a nonperturbative instanton effect, exactly as in the $N=2$ Yang-Mills case. Thus the idea of a dual formulation of string theory would dovetail nicely with the observations of this paper.

Related phenomena were discussed in [32]. In [33], an Abelian Higgs model, which has soliton strings, was coupled to gravity. Instantons were found corresponding to a soliton string worldsheet wrapping around a black hole horizon. These instantons give nonperturbative corrections for example to the Hawking temperature. The strength of these effects goes as

$$
e^{-T A / \hbar}
$$

where $T$ is the soliton string tension and $A$ is the area of the horizon. In [32] the same effect was considered in the context of fundamental string theory. In this case the instantons are fundamental string worldsheets wrapping around a black hole horizon, i.e. worldsheet instantons. The strength of the effects goes as

$$
e^{-A / 2 \pi \alpha^{\prime}}
$$

This expression does not involve $\hbar$ and so is viewed as a classical effect. However (4.5) reduces to (4.4) when reexpressed in terms of the string tension $T=\hbar / 2 \pi \alpha^{\prime}$. Similarly the worldsheet instantons which produce type IIA conifold singularities become ordinary spacetime instantons when the worldsheet is itself a soliton. 


\section{Conclusions}

In closing we mention that our analysis has brought to light several interesting features of string theory whose significance goes beyond the example discussed here:

1. Nonperturbative quantum effects of a certain restricted type may occur in string theory which are not suppressed as $g_{s} \rightarrow 0$.

2. Classical string theory is inconsistent without the inclusion of such effects.

3. The quantum consistency of string theory requires that extremal black holes be treated as elementary quanta.

\section{Acknowledgements}

I am grateful to K. Becker, M. Becker, P. Candelas, B. Greene, J. Harvey, D. Lowe, E. Martinec, J. Polchinski, N. Seiberg, L. Thorlacius and M. Srednicki for useful discussions. This work was supported in part by DOE grant DOE-91ER40618.

\section{Appendix A. Superpotentials}

In this appendix we consider the behavior of a superpotential near a conifold. We consider an $N=1$ theory with moduli fields parameterizing a space with a conifold singularity. This arises in Calabi-Yau compactification of heterotic string theory.

The superpotential $W$ is a section of a line bundle $L$ whose curvature is the Kahler form [34]. $L$ has no monodromy about a conifold singularity and $\mathcal{K}$ is finite there. A generic superpotential can therefore be expanded about a conifold singularity at $Z^{1}=0$ as

$$
W=w_{0}+w_{1} Z^{1}+\ldots
$$

The scalar potential is related to $W$ by

$$
V=e^{\mathcal{K}} \mathcal{G}^{I \bar{J}} D_{I} W D_{\bar{J}} \bar{W}-3 e^{\mathcal{K}} W \bar{W}
$$

Near the conifold the inverse metric behaves as

$$
\mathcal{G}^{1 \overline{1}} \sim \frac{1}{\ln \left(Z^{1} \bar{Z}^{1}\right)}+\ldots
$$

$V$ will then behave as

$$
V \sim V_{0}+\frac{w_{1}^{2}}{\ln \left(Z^{1} \bar{Z}^{1}\right)}+\ldots .
$$

which has a sharp minimum at $Z^{1}=0$. Thus it is plausible that a superpotential could have local minima at conifold singularities.

Of course the $N=1$ supersymmetry present in heterotic string theory allows perturbative corrections to $\mathcal{G}$. These could qualitatively alter the geometry near the conifold, so these observations should be regarded as merely suggestive. 


\section{References}

[1] P. Candelas, X. de la Ossa. P. Green and L. Parkes, "A Pair of Calabi-Yau Manifolds as an Exactly Soluble Superconformal Field Theory" Nucl. Phys. B359 (1991) 21.

[2] P. Candelas, P. Green and T. Hubsch, "Rolling Among Calabi-Yau Vacua", Nucl. Phys. B330 (1990) 49.

[3] P. Candelas and X. de la Ossa, "Comments on Conifolds", Nucl. Phys. B42 (1990) 246.

[4] P. Candelas and X. de la Ossa, "Moduli Space of Calabi-Yau Manifolds", Nucl. Phys. B42 (1990) 246.

[5] P. Aspinwall, B. Greene and D. Morrison, B416 (1994) 414.

[6] B. Greene, "Lectures on Quantum Geometry", to appear.

[7] G. Horowitz and A. Strominger, "Black Strings and p-branes," Nucl. Phys. B360 (1991) 197.

[8] N. Seiberg and E. Witten, "Electromagnetic Duality, Monopole Condensation and Confinement in N=2 Supersymmetric Yang-Mills Theory", hep-th/9407087, Nucl. Phys. B426, (1994), 19.

[9] M. Dine, P. Huet, and N. Seiberg, "Large and Small Radius in String Theory", Nucl. Phys. B322 (1989) 2073.

[10] C.M. Hull and P.K. Townsend, "Unity of Superstring Dualites", QMW-94-30, $\mathrm{R} / 94 / 33$, hep-th/9410167.

[11] E. Witten, "String Theory Dynamics in Various Dimensions", hep-th/9503124.

[12] A. Ceresole, R. D'Auria, S. Ferrara and A. Van Proeyen, "Duality Transformations in Supersymmetric Yang-Mills Theory Coupled to Supergravity, "hep-th/9502072.

[13] A. Sen, "String-String Duality Conjecture in Six Dimensions and Charged Solitonic Strings", hep-th/9504027.

[14] J. Harvey and A. Strominger, "The Heterotic String is a Soliton", hep-th/9504047.

[15] S. Shenker, "The Strength of Nonperturbative Effects in String Theory", in proceedings of the Cargese Workshop on Random Surfaces, Quantum Gravity and Strings (1990).

[16] B. de Wit, P. Lauwers and A Van Proeyen, "Lagrangians of N=2 Supergravity-Matter Systems", Nucl. Phys. B255 (1985) 269.

[17] A. Strominger, "Special Geometry", Comm. Math. Phys. 133 (1990) 163.

[18] G. Tian, in Mathematical Aspects of String Theory, ed. S. T. Yau, World Scientific (1987) 629.

[19] J. H. Schwarz, Nucl. Phys. B226 (1983) 269.

[20] M. J. Duff and J. X. Lu, "The Self-Dual Type IIB Superthreebrane", Phys. Lett. 273 (1991) 409. 
[21] P. Breitenlohner, D. Mason and G. Gibbons, "4-Dimensional Black Holes from KaluzaKlein Theories", Comm. Math. Phys. 120 (1988) 295.

[22] N. Seiberg, "Observations on the Moduli Space of Superconformal Field Theories", Nucl. Phys. B303, (1986), 288; P. Aspinwall and D. Morrison, "String Theory on K3 Surfaces", preprint DUK-TH-94-68, IASSNS-HEP-94/23, hep-th/9404151.

[23] S. Cecotti, S. Ferrara and L. Girardello, "Geometry of Type II Superstrings and the Moduli of Superconformal Field Theories", Int. J. Mod. Phys. 4 (1989) 2475.

[24] G.W. Gibbons and C.M. Hull, Phys. Lett. 109B (1982) 190.

[25] C. G. Callan, J. A. Harvey and A. Strominger, "Worldbrane Actions for String Solitons," Nucl. Phys. B367 (1991) 60.

[26] K. Becker, M. Becker and A. Strominger, unpublished.

[27] A. Dabholkar and J. A. Harvey, "Nonrenormalization of the Superstring Tension, " Phys. Rev. Lett. 63 (1989) 719.

[28] A. Dabholkar, G. Gibbons, J. A. Harvey and F. R. Ruiz, "Superstrings and Solitons, " Nucl. Phys. B340 (1990) 33.

[29] A. Strominger, "Heterotic Solitons," Nucl. Phys. B343 (1990) 167; E: Nucl. Phys. B353 (1991) 565.

[30] M. J. Duff and J. X. Lu, "Remarks on String/Fivebrane Duality," Nucl. Phys. B354 (1991) 129; "String/Fivebrane Duality, Loop Expansions and the Cosmological Constant," Nucl. Phys. B357 (1991) 534.

[31] M. J. Duff, "Strong/Weak Coupling Duality from the Dual String", NI-94-033, CTPTAMU-49/94, hep-th/9501030.

[32] S. Giddings, J. Harvey, J. Polchinski, S. Shenker and A. Strominger, hep-th/9309152, Phys. Rev. D50 (1994) 6422.

[33] S. Coleman, J. Preskill and F. Wilczek, Phys. Rev. Lett. 67, (1991) 1975; Nucl.Phys. B378 (1992) 175.

[34] J. Bagger and E. Witten, Nucl. Phys. B222 (1983) 1. 